\title{
Turning the actin nucleating compound miuraenamide into nucleation inhibitors
}

Shuaijun Wang ${ }^{1 \S}$, Maximilian Meixner²§, Lushuang $\mathrm{Yu}^{1}$, Ling Zhuo ${ }^{1}$, Lisa Karmann ${ }^{3}$, Uli Kazmaier ${ }^{3}$, Angelika M. Vollmar ${ }^{1}$, Iris Antes ${ }^{2 *}$, and Stefan Zahler ${ }^{1 *}$

${ }^{1}$ Department of Pharmacy, Ludwig-Maximilians-Universität, 81377 Munich, Germany

${ }^{2}$ Computational Chemical Biology, Technische Universität München, TUM School of Life Sciences, Emil-Erlenmeyer-Forum 8, 85354 Freising and Center for Protein Assemblies (CPA), Ernst-Otto-Fischer Str. 8, 85747 Garching, Germany

${ }^{3}$ Organic Chemistry, Saarland University, 66123 Saarbrücken, Germany

§shared first authors

${ }^{*}$ corresponding authors

Supporting Information 


\section{Supplementary text 1 :}

\section{$\underline{\text { LK701-actin monomer }}$}

From the docking calculations in the actin monomer, all generated poses were clustered and all poses inside each cluster were ranked with the interaction score of DynaDock (see Experimental Procedures in the main text) and the best scored poses of the three highest populated clusters ( $A^{\text {mono }}, B^{m o n o}$ and $C^{\text {mono }}$ in the following, black in Figures S1A, S1C and S1E, respectively) were further investigated by simulating the dynamics for 200 ns in three independent molecular dynamics (MD) simulations each. For each selected pose, the three replica simulations were combined and clustered and the representative structure of the highest populated cluster was taken as the most dominant structure of the simulations and as the equilibrated pose of $A^{\text {mono }}, B^{\text {mono }}$ and $C^{\text {mono }}$, respectively (orange in Figures S1A, S1C and S1E). Detailed analysis of each simulation revealed that the protein is very well equilibrated in the presence of the ligand (blue lines in Figures S1B, S1D and S1F). For the analysis of the ligand dynamics of each selected pose, the root-mean square deviation (RMSD) of the position of LK701 heavy atoms throughout each simulation (Rep 1-3) was calculated relative to the docked position (black lines in Figures S1B, S1D and S1F).

For $A^{\text {mono }}$ (Figures S1A and S1B), the MD simulations revealed a stable position of LK701 in replica 2 and 3 indicated by low ligand RMSD values between 3-5 $\AA$ from the docked position. The same position is initially found in replica 1 for the first 50 ns of the simulation followed by larger structural rearrangements of the ligand inside the binding site due to the open and surface-exposed nature of the macrolide binding cleft.

Large fluctuations of LK701 compared to the docked position of the second selected docking pose $\mathrm{B}^{\text {mono }}$ (Figure S1C) were observed during all replica simulations of that pose (black lines in Figure S1D). In replica 3 the ligand stayed at a position far from the docked location (8 $\AA$ ), while in replica 1 and 2 it moved even further away, indicated by increasing RMSD values throughout the simulations. Thus, the position of $\mathrm{B}^{\text {mono }}$ was unstable as it could not be reproduced in independent long-term MD simulations.

The ligand lied half-outside the binding site in the third selected docking pose $\left(\mathrm{C}^{\text {mono }}\right.$, black in Figure S1E) and the dynamics of this pose revealed that it stayed very close to that position ( $2 \AA)$ during most of the simulated time in replica 1 and 3 (Figure S1F). Only in replica 2 the ligand moved away from its docked position, leaving the binding site. Although the docked pose could be reproduced independently in two of the replicas, the docked position did not match the macrolide binding cleft (see position of miuraenamide A for comparison, gray in Figure S1E) and the ligand did not move further into the binding site during the simulations. 
Summarizing the results of the MD simulations of the LK701-actin monomer complexes, it can be stated that a stable conformation inside the macrolide binding cleft could only be formed by $A^{\text {mono }}$, which could be obtained independently in different simulations, while for $B^{\text {mono }}$ and $\mathrm{C}^{\text {mono }}$ no stable positions inside the binding cleft were found. Therefore, the final equilibrated binding pose of LK701 in the actin monomer LK701 mono was determined as the representative structure of the highest populated cluster obtained from all combined simulations of $A^{\text {mono }}$ (orange in Figure S1A). Comparing the final position of LK701 to the predicted binding pose of miuraenamide $A^{1}$ (gray sticks in Figure S1A) shows that LK701 lies deeper inside the macrolide binding cleft.

\section{LK701-actin nucleus}

Similar to the docking in the actin monomer, three docking poses of LK701 in the actin nucleus $A^{\text {tri }}$ (best scored pose of the highest populated cluster of all docked poses), $B^{\text {tri }}$ (closest docking pose to the predicted binding position in LK701 ${ }^{\text {mono }}$ ) and $\mathrm{C}^{\text {tri }}$ (second closest docking pose to the predicted binding position in LK701 ${ }^{\mathrm{mono}}$ ) were selected for which further MD simulations were performed. The analyses of those simulations are shown in Figure S2. Looking at all docked poses (data not shown) it was clear that the DNase-I binding loop (D-loop) of subunit $n+2$ was blocking the ligand from proper binding to the macrolide binding cleft since no poses were entirely located in the binding site after refinement. Most of the poses were located behind the binding site (exemplary shown for $A^{\text {tri }}$ in black in Figure S2A), while the remaining poses were located on the solvent exposed side of the subunit, closer to the equilibrated binding position found in the actin monomer (exemplary shown for $B^{\text {tri }}$ and $C^{\text {tri in }}$ black in Figures S2D and S2G, respectively).

As shown for the most dominant position of LK701 of the MD simulations of $A^{\text {tri }}, B^{\text {tri }}$ and $C^{\text {tri }}$ (orange in Figures S2A, S2D and S2G, respectively), LK701 did not move closer to the predicted position in LK701 ${ }^{\text {mono }}$ (green) compared to the initial docking position (black) and the D-loop of the adjacent subunit obtained a similar conformation inside the macrolide binding cleft (cyan) independent of the location of the ligand, emphasizing the strong longitudinal interaction between subunits in the nucleus. This is underlined by the respective secondary structure (DSSP) analysis for residues of the D-loop of subunit $n$ in Figures S2B, S2E and $\mathbf{S 2 H}$, that indicate that the backbone of the core-residues of this loop was in a bend or turn conformation for most of the simulated time and did not change its secondary structure type throughout the simulation, which proves a stable loop conformation. Figures S2C, S2F and S2I show the RMSD analyses of different regions of the complex. The overall protein backbone (blue) does not fluctuate much in RMSD, indicating a well equilibrated system in all three cases. Even the RMSD of the position of the D-loop backbone atoms (green) show only small oscillation around a constant value proving once more the stability of this formation. 
Interestingly, the RMSD of the position of LK701 (black) with respect to the predicted LK701 mono reference position shows that for $A^{\text {tri }}$ and $C^{\text {tri }}$ (Figures S2C and S2I) the ligand stayed roughly at the same position outside of the binding cleft, while for B ${ }^{\text {tri }}$ (Figure S2F), the ligand moved even further away from the binding site throughout the simulation.

These results strongly suggest that LK701 cannot enter the macrolide binding cleft while the D-loop of the adjacent subunit is bound; furthermore, that this loop is forming a strong interaction across the longitudinal axis of the forming filament and that - in this arrangement LK701 cannot form a stable complex with the actin nucleus.

\section{Comparison between the actin binding modes of LK701 and miuraenamide A}

As stated above, LK701 is located deeper in the macrolide binding cleft compared to the parent compound miuraenamide A. Aiming for a structural explanation of the observed experimental differences, the equilibrated binding positions of both compounds (LK701mono and the predicted binding position of miuraenamide $A^{1}$, see Figure S1) were thoroughly investigated for their protein ligand interactions. Figure S3 shows an overview of the interactions predicted by PoseView $^{2}$ in a $2 \mathrm{D}$ sketch, as well as transferred onto a three-dimensional representation of the poses highlighting the most important amino acid residues and their type of interaction. The macrolide binding cleft is mainly dominated by hydrophobic residues (see top of Figures S3A and S3B) that form interactions with the hydrophobic regions of the macrocycle and the phenyl side extensions of both compounds. In the case of miuraenamide bound to the actin trimer, the macrolide binding cleft is located at the inter-subunit interface of subunit $n$ (cyan) and $n+2$ (light blue) and the compound, thus, interacts with residues of both subunits. Importantly, hydrogen bonds are formed with residues from subunit $n$, which promote the crucial rearrangement of the D-loop of that subunit as discussed in the previous study 1 . Additionally, the bulky aromatic side extensions of miuraenamide increase its steric hinderance, keeping it from binding deep into the macrolide binding cleft of subunit $n+2$. In contrast, LK701, bound to the actin monomer and therefore lacking interactions with an adjacent subunit as well as the second aromatic side extension, fills the macrolide binding cleft completely. The position of the macrocycle is slightly rotated compared to the bound miuraenamide and, thereby, the phenyl side extension of LK701 fits into a small hydrophobic sub-pocket formed by $\mathrm{Tyr}_{143}$, $\mathrm{Leu}_{346}, \mathrm{Phe}_{352}, \mathrm{Leu}_{349}$ and $\mathrm{Met}_{355}$, forming $\pi-\pi$ stacking interactions with $\mathrm{Phe}_{352}$ at the tip of that pocket (top right in Figures S3B and S3D). Because of that, the hydrogen bond acceptor oxygen atoms of the macrocycle, which form hydrogen bridges across actin subunits in the miuraenamide bound actin trimer, are shielded by the entrance of the macrolide binding cleft and therefore less solvent exposed. Besides LK701 blocking the macrolide binding cleft, this could be a possible reason why an additional subunit cannot bind to the LK701-bound actin monomer, thereby inhibiting the proper nucleation of actin. 


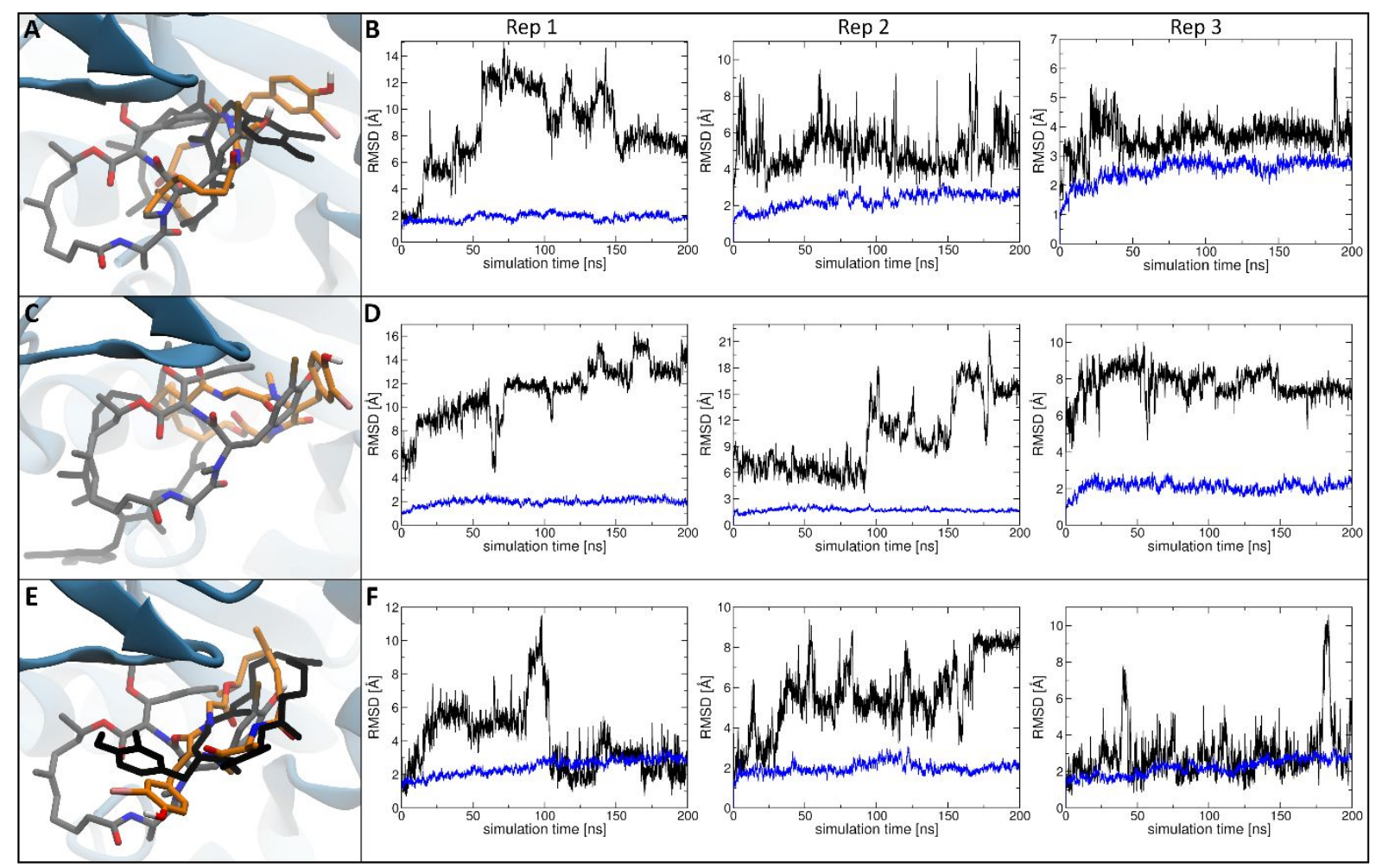

Figure S1: Molecular dynamics simulations of three docking poses of LK701 in the actin monomer. A, $C$ and E: final equilibrated pose of LK701 (orange) from the simulations of the best ranked poses of the three highest populated clusters of docking poses $A^{\text {mono }}, B^{\text {mono }}$ and $C^{\text {mono }}$ (black), respectively, compared to the position of miuraenamide $A$ (gray) for orientation. $B, D$ and F: root-mean square deviation (RMSD) of protein backbone heavy atoms (blue) with respect to the first frame and RMSD of the position of ligand heavy atoms (black) relative to the docked pose corresponding to those shown in black in $A, C$ and E, respectively, throughout 3x200 ns of molecular dynamics simulations (Rep 1-3). 


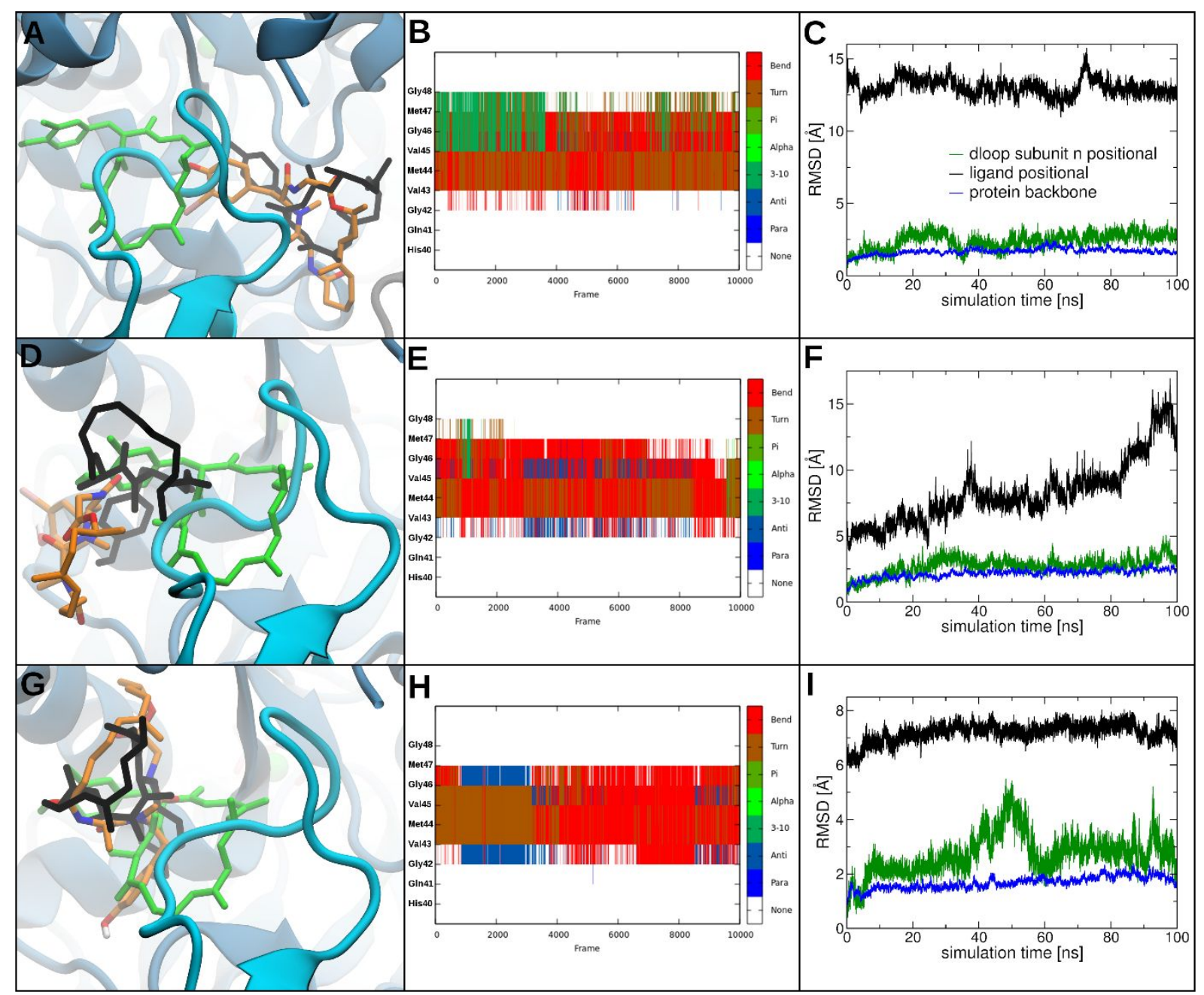

Figure S2: Molecular dynamics simulations of three selected docking poses of LK701 in the actin trimer nucleus: $A, D$ and G: equilibrated position of LK701 (orange) from MD simulations of the best ranked structure of the highest populated cluster of docking poses $\left(A^{\text {tri }}\right)$, closest $\left(B^{\text {tri }}\right)$ and second closest docking pose $\left(C^{t r i}\right)$ with respect to the predicted binding position in the actin monomer (black), respectively. Predicted position of LK701mono shown in green for reference. $B, E$ and $H$ : secondary structure analysis of the D-loop of subunit $n+2$ throughout the $M D$ of $A^{t r i}, B^{t r i}$ and $C^{t r i}$, respectively. C, $F$ and I: root-mean square deviation (RMSD) of protein backbone heavy atoms (blue) throughout the simulation, RMSD of the position of LK701 heavy atoms relative to the predicted binding position in the actin monomer (black) and RMSD of the position of D-loop backbone heavy atoms with respect to the first frame of the simulation (green), key shown in $C$ applies to $F$ and $I$. 


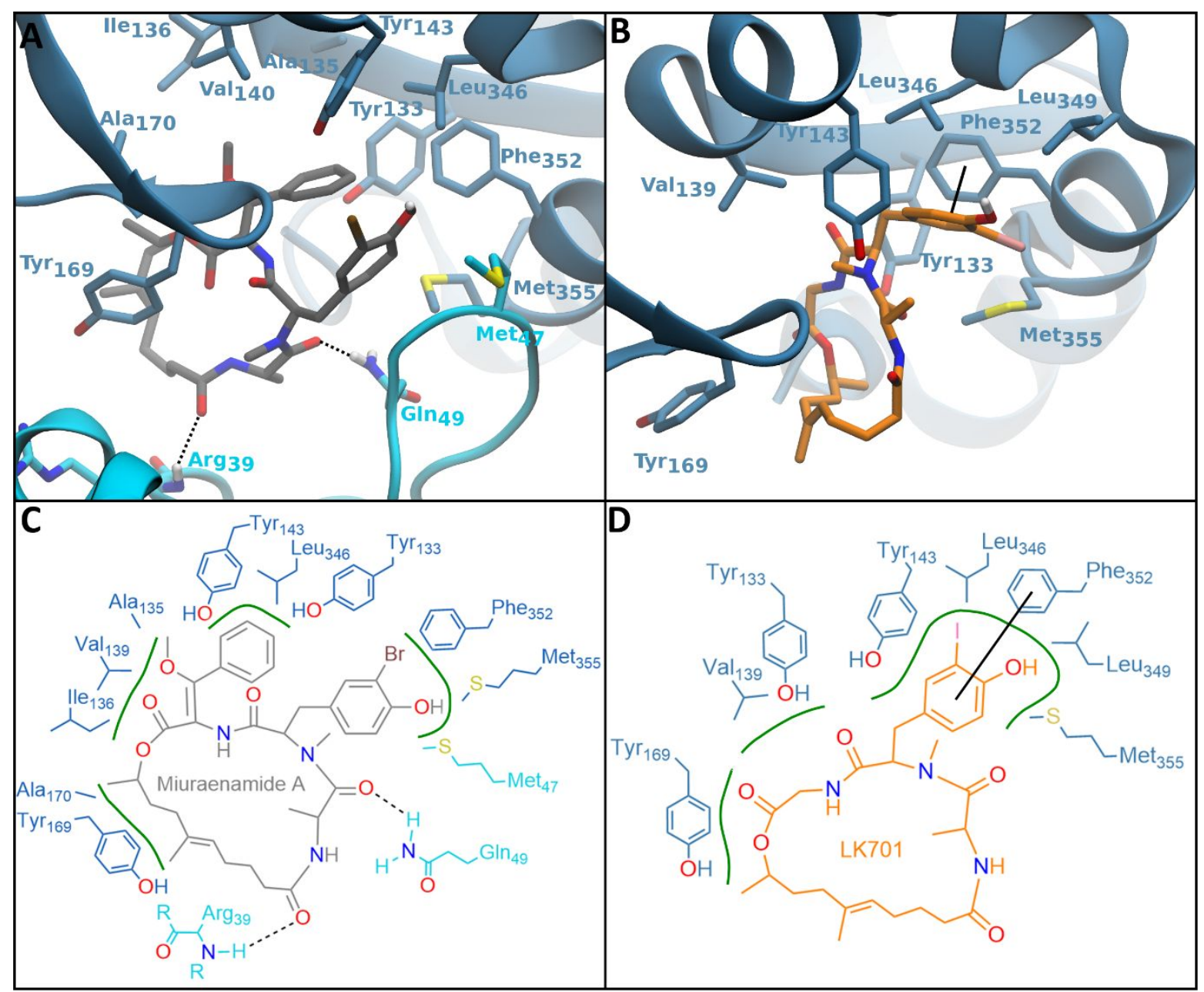

Figure S3: Overview of protein ligand interactions comparing miuraenamide $A(A: 3 D$ and $C: 2 D)$ with LK701 (B: $3 D$ and D: 2D). The interactions were calculated from the equilibrated binding poses of miuraenamide $A$ in the bound actin trimer ${ }^{1}$ and LK701 in the bound actin monomer with PoseView ${ }^{2}$. In (A) and (B) the actin protein is shown as cartoon, the ligands and the relevant amino acids that form protein ligand interactions in sticks (color code according to Figure 3, additionally: yellow (sulfur), brown (bromine), other (carbon)). Polar hydrogen atoms on protein shown only for those involved in hydrogen bridging. Protein ligand interactions are displayed as: hydrogen bonds (dashed lines), $\pi-\pi$ stacking (solid black lines) and hydrophobic interactions (in $C$ and D: solid green lines). Amino acid numbering in accordance with the actin monomer published by Kabsch and coworkers ${ }^{3}$. 
1. Wang, S.; Crevenna, A. H.; Ugur, I.; Marion, A.; Antes, I.; Kazmaier, U.; Hoyer, M.; Lamb, D. C.; Gegenfurtner, F.; Kliesmete, Z., Actin stabilizing compounds show specific biological effects due to their binding mode. Scientific reports 2019, 9 (1), 1-17.

2. $\quad$ Stierand, K.; Maass, P. C.; Rarey, M., Molecular complexes at a glance: automated generation of two-dimensional complex diagrams. Bioinformatics 2006, 22 (14), 1710-6.

3. Kabsch, W.; Mannherz, H. G.; Suck, D.; Pai, E. F.; Holmes, K. C., Atomic structure of the actin: DNase I complex. Nature 1990, 347 (6288), 37-44. 